\title{
Dysautonomia: A Novel Approach to Understanding of Vasculitis and Type Ii Diabetes
}

\author{
Ramesh K. Adiraju \\ RENU-CA Research Institute, P.C, 501 Bath Road Bristol, PA 19007
}

Received: 12 April, 2017; Accepted: 21 September, 2017; Published: 25 September, 2017

*Corresponding author: Ramesh K Adiraju, RENU-CA Research Institute, 501 Bath Road Bristol, PA 19007, Tel: 215-785-9578; Fax: 215-7859579; email: renu-ca@comcast.net

\begin{abstract}
Understanding of the atherosclerosis process has evolved from simple plaque build-up causing blockage to inflammation and endothelial dysfunction. The underlying etiology for endothelial dysfunction and vascular inflammation is autonomic imbalance. Autonomic imbalance also causes hormonal dysregulation and cellular receptor sensitivity. Therefore, dysautonomia is the underlying etiology behind type 2 diabetes and vascular inflammation that predispose to atherosclerosis. Regulating dysautonomia is a more definitive and long-term solution to effectively treat type 2 diabetes and vascular atherosclerosis.
\end{abstract}

Keywords: Dysautonomia; Vasculitis; Diabetes type2; Inflammation; Atherosclerosis

\section{Introduction}

Vascular atherosclerosis, like type 2 diabetes, is a relentless systemic process. Despite identification of several risk factors and major progress in risk factor interventions and management, the progression of vascular disease process and its related complications continue to be at the forefront for mortality and morbidity in medicine and a major contributor for the cost of healthcare services. Because of suboptimal effect of current standard risk factor interventions in preventing the progression and elimination of vascular disease, there has been extensive research with committed dedication for vascular biology research over the past two decades. Vascular endothelial dysfunction and vascular inflammation have emerged as key underlying pathophysiologic derangements that propagate systemic, diffuse vasculopathy, particularly in type 2 diabetes patients and women. Vascular pathology and disease progression is also uniquely different in type 2 diabetes with diffuse involvement and more fibrosis than atherogenesis. This is a pattern that is also noted in vascular disease in women. Metabolic syndrome and sleep apnea syndrome have been identified as a cluster of conditions where vascular disease manifestations are a common consequence. There is increasing awareness about the clustering of hypertension, hypertriglyceridemia, obesity and type 2 diabetes with metabolic syndrome and sleep apnea syndrome. Systemic vascular inflammation with elevated inflammatory markers, hsCRP, homocysteine (14) have been identified as predictors of vascular disease progression in these clusters. These observations have led to, in recent years, clinical trials studying the effect of treating systemic inflammation in the prevention and progression of vascular disease (CIRT).

\section{Vascular Circulatory System}

The circulatory system is the most important system in the body. While the vascular system is a closed-circuit system comprising of delivery (arterial) and clearing (venous) channels, the circulatory system has the dual role of being a protective system and a transport mechanism for hormones, oxygen, nutrients, and metabolically active substances throughout the body. It also functions as a clearing system for metabolic waste products such as lactic acid produced by the body through the venous system. This is an active process and in humans who are upright this involves active pulsatile pumping by the peripheral venous system. Humans through the process of evolution became upright, bi-pedal beings. Bi-pedal posture mandates critical optimal peripheral a-v capillary tone - THE PITCH TONE and active pumping mechanism by the peripheral venous system to maintain intravascular volume and cerebral perfusion. The saphenous venous system of the calf is therefore referred to as the second heart pump (Guyton physiology).Optimal peripheral a-v capillary tone is therefore essential to maintain systemic vascular tone - blood pressure, vascular homeostasis and cardiovascular system stability. PITCH TONE is a neglected essential physiologic link between peripheral vasculature and brainstem that is crucial for balanced functioning of the autonomic nervous system. Pitch tone is maintained by a balanced Sympathetic Nervous System (SNS) and parasympathetic (PSNS) input. This is achieved by a steady basal SNS tone and a dynamic modulation of the SNS oscillations by the PSNS. Loss of pitch tone induces a hypothalamic response at the brainstem that in turn triggers a Central Vagal Nucleus (CVN) response at the brainstem. CVN has reflex feedback inhibition of the hypothalamus and SNS stimulation triggering a hyper-sympathetic state. This is called paradoxic parasympathetic syndrome (PPS) (Adiraju). PPS can have multi organ system effects due to regulatory function of the CVN on the cardiovascular, 
hormonal, and immune systems. Heart and vascular system have heavy autonomic innervations through cardiac autonomic ganglia and vasa-nervosa. Hormonal regulation, particularly insulin and thyroid hormones, is coordinated by the autonomic system. Type 2 diabetes mellitus, secondary hypothyroidism and vitamin $\mathrm{D}$ deficiency that is commonly associated with these disorders along with the pathophysiology underlying atherosclerotic vascular disease is, therefore, a consequence of PPS and dysautonomia. Systemic inflammation is also triggered by PPS, hence the increasing evidence for inflammatory markers such as hsCRP and homocysteine in cardiovascular disease and Type2 diabetes vasculopathy. Diabetic vasculopathy demonstrates more fibroblast reaction with fibrosis and increased intramural PAI synthesis resulting in hyperplasia and hypercoagulable state.

There are two essential structures for the circulatory systemthe vascular endothelium and peripheral a-v capillary network. Optimal vascular system integrity, vaso-reactivity and vascular homeostasis are dependent on two important factors: 1) Vascular endothelial membrane integrity 2) Optimal Peripheral capillary tone-The Pitch Tone.

\section{Vascular Endothelium}

Vascular endothelium is a unique structure. It is a multitasking organ that has protective and physiologically active functions. As a physiologically active organ, it maintains Local Vascular Homeostasis (LVH). As a protective organ it forms an effective barrier for the vascular wall to prevent inflammation and injury from chemical and free radical exposure of the subendothelium and prevents chemotaxis and smooth muscle and fibroblast migration from the sub-endothelium. Vascular endothelium is built uniquely to accomplish these functions. Vascular endothelium comprises of a single layer of cells linked by gap junctions (smooth muscle bands) which are regulated by autonomic nervous system through vasa nervosa. Vascular endothelial membrane acts as an impermeable barrier as well as active absorptive/secretory transport membrane through regulation of gap junctions (FIG-I). Vascular endothelial cells also produce local hormones such as EGF, VGDF, Endothelin, and regulate vascular wall Nitric Oxide (NO) concentration. Optimal vascular NO concentration determines the arterial tone and vasodilation. Vascular NO production is through Soluble Guanylate Cyclase (SGC) via Cyclic GMP (C.GMP) pathway. Optimal balance between pro-oxidative (Reactive oxygen species/free radicals) and anti-oxidant pool in the vascular wall facilitates normal S. GC/CyGMP pathways for NO synthesis and maintains normal vasoreactivity i.e. vasodilation to acetyl choline stimulation. Peripheral A-V capillary tone as noted above also plays an important role in maintaining the vascular homeostasis in bi-pedal state.

\section{Maintaining Pitch Tone and endothelial integrity is dependent on several systemic and local factors}

Systemic factors: Hormones - catechol amines, thyroid hormones, growth hormone, vasoactive hormones such as vasopressin and renin angiotensin system. There are also other regulating factors such as serum leptin levels, Immune system reactions and coagulation factors.

Local factors: Vascular nitric oxide concentration, antioxidants-superoxide dismutase and catalase, reactive oxygen species (ROS), prooxidative hormones- endothelin, angiotensin-II, and kallikrin/kinin system, local TPA to PAI balance, interleukins and prostaglandins.

\section{Functions of vascular endothelium}

- Active exchange of oxygen, essential and nutrient substances and metabolites transported in blood stream with body tissues and organs.

- Synthesis, release and transport of vasoactive chemicals and -local hormones by active process of secretion and absorptionInterleukins, prostaglandins-PGE, PGI, Prostacyclin. These substances along with No generated through soluble guanylate cyclase/cGMP pathway are responsible for vascular reactivity, endothelial integrity and protection from vascular inflammation.

- Active regulation of local vascular hormones-EDGF, PDGF, endothelin, optimal balance between vascular wall anti-oxidant to pro-oxidative pool to maintain vascular homeostasis.

- Protective membrane for vascular wall and sub-endothelium to prevent chemotaxis, mast cell and smooth muscle migration and proliferation with endothelial and vascular inflammation.

Vascular endothelium achieves these goals through the process of dynamic variable permeability and barrier functions through modulation of vascular endothelial cell GAP junction's contractility. GAP junctions, like sphincters and gastro-intestinal wall smooth muscle, are made-up of involuntary smooth muscle bands that are regulated by balanced sympathetic and parasympathetic input through vasa-nervosa (Figure-I). Timely contraction and relaxation of the GAP junction smooth muscle bands is brought about by balanced sympathetic and parasympathetic stimulation. This phenomenon is similar to the involuntary smooth muscle functions such as peristaltic movements of the gastrointestinal tract and myocardial contractility.

Peripheral venous system plays as important a role as the arterial system in maintaining an optimal and healthy cardiovascular system in humans. Upright posture of humans, as noted before mandates an active peripheral venous pump and optimal Pitch tone to maintain intravascular volume and cerebral circulation. Pitch tone is maintained by a constant baseline sympathetic tone at half strength with an overriding dynamic balanced sympathetic and parasympathetic input via vasa nervosa (Figure).

Maintaining the integrity of the vascular endothelium allows normal physiologic reactivity of the vascular system to autonomic stimulation and prevents vascular inflammation. This is a function of the autonomic nervous system in optimal balance. 
Auto regulation of cellular physiology and cellular receptor activity is also a function of balanced autonomic nervous system. Therefore, maintaining balanced autonomic nervous system input is essential for vascular and endothelial integrity and local vascular homeostasis. Balanced autonomic nervous system also ensures hormone regulation, cardio-vascular system and immune system stability. Abnormal parasympathetic input triggers a hyper-sympathetic state and predisposes to type 2 diabetes and cardio-vascular disease progression. GAP junction dysregulation due to dysautonomia triggers vascular endothelial dysfunction, hypercoagulable state, dysfunctional local vascular homeostasis and abnormal vascular reactivity. Abnormal vascular reactivity is paradoxic vasoconstriction to acetyl choline stimulation during Brachial Artery Reactivity Testing (BART). This is a well-documented phenomenon observed in atherosclerosis particularly in type 2 diabetes and vascular inflammation. Laxity of GAP junction smooth muscle bands due to dysregulation by the autonomic vasa nervosa leads to exposure of vascular wall sub-endothelium to luminal chemicals that trigger vascular injury and chemotaxis. Macrophage and smooth muscle cell migration, altered local prostaglandins, interleukins and reactive oxygen species along with chemotaxis leads to abnormal local vascular homeostasis and inflammation causing endothelial dysfunction and vasculitis. Omega-3 free fatty acid molecules interact with activated macrophages and are modified to EFOX (COX-2 (cyclooxygenase) dependent Electrophile Oxo-derivative molecules). EFOX molecules trigger many cellular physiologic changes that have anti-oxidant and anti-inflammatory effects through their interaction with cox- 1 and cox- 2 enzymes.

Cox-I is present constantly in tissues and cells and is responsible for essential functions such as cellular receptor sensitivity and mucous membrane integrity and contributes to maintaining GAP junction function. Cox-II is only released in response to hypoxic stress, toxin exposure, growth factors, cytokines and other stress stimuli. Cox-II enhances the formation of prostaglandins that mediate pain and inflammation. Cox-II inhibitors therefore help alleviate pain and inflammation without blocking Cox-I but in the process make the body vulnerable to endogenous stresses that predispose to increase in vascular complications.

\section{GAPJUNCTIONS MODULATION \& DYNAMIC VASCULAR ENDOTHELIAL PERMIABILITY BY AUTONOMIC VASANERVOSA}

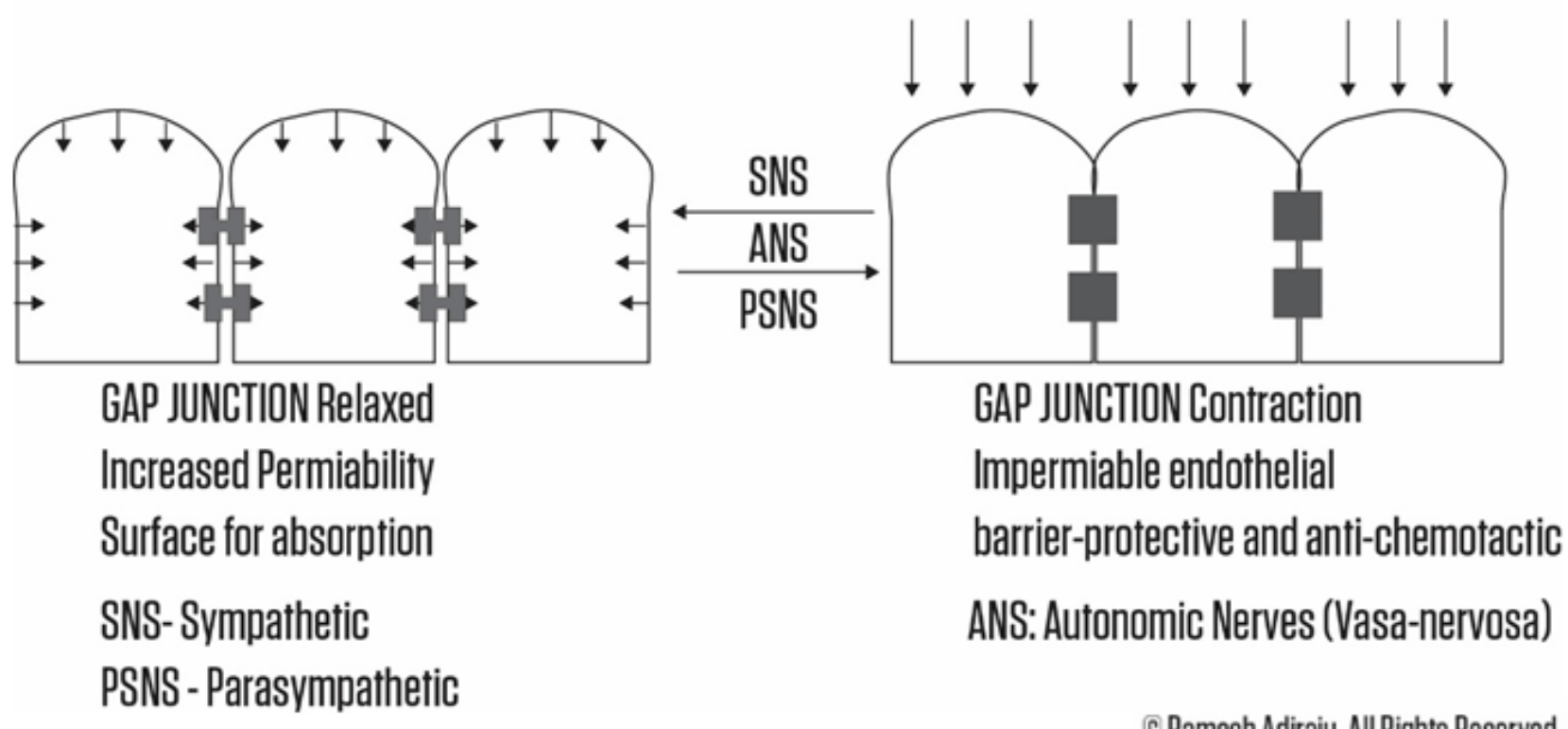

(C) Ramesh Adiraju. All Rights Reserved

Figure 1: 
Cell membrane eicosanoids - arachidonic acid and Eicosa Pentanoic Acid (EPA) form biologically active eicosanoids that promote vascular reactions and inflammation such as: Prostaglandins (PHZn)-inflammation and Immune suppression, thromboxane (TXZn) thrombosis, prostacyclins-Hypotesion and pain, lipoxins- vascular reactivity, anti-inflammation and Leukotrienes-chemotaxis. These eicosanoids are released locally and are regulated by autonomic nervous system via cell membrane receptor regulation and cellular physiology in response to stress. Studies in northern Europe and USA have established that the incidence of coronary heart disease mortality is 2.5 times higher for people with cholesterol in the highest $25^{\text {th }}$ percentile and lowest $25^{\text {th }}$ percentile. For the same cholesterol level, a person in west Scotland has 8 times more likely mortality from coronary heart disease than a person in Catalonia Spain, or a person on Mediterranean diet. Therefore, cholesterol is a contributing factor in atherosclerotic vascular disease but lowering cholesterol has not been shown to reduce mortality. This is because endothelial dysfunction with vascular inflammation is the underlying mechanism for atherogenesis. Metabolic dysfunction causes lipid abnormality that is a contributor to atherosclerosis in a setting of vulnerable endothelium. Endothelial dysfunction and abnormal peripheral Pitch Tone propagate progression of atherosclerosis and cardiovascular disease state. (Figure-2, Figure-3)

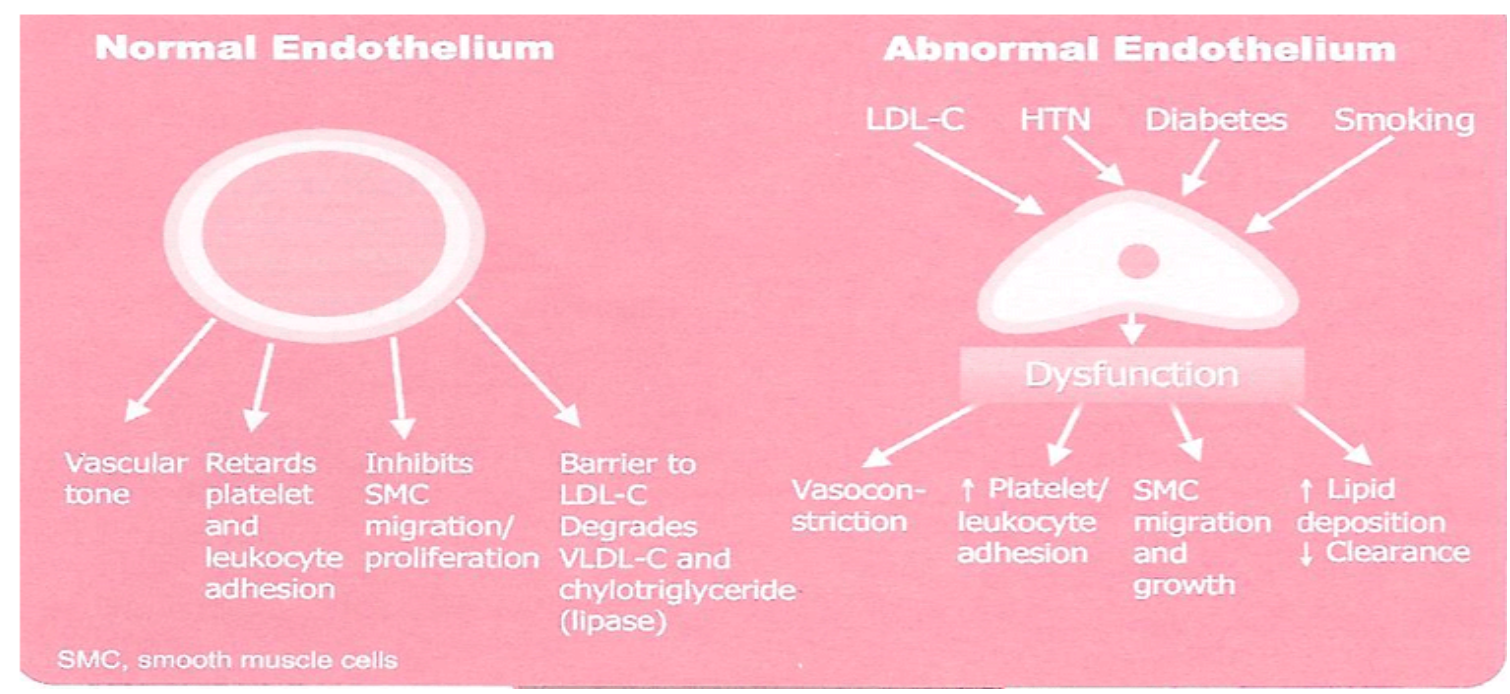

Figure 2: Courtesy Dzau VJ, Gibbons GH, Cook JP et al, Vascular biology and medicine in the 1990s: scope, concepts, potentials, and perspectives. Circulation, 1993;87:705-719.

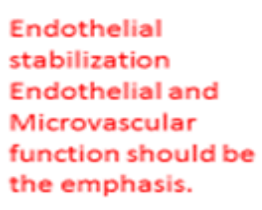

Figure 3: Courtesy: EndotheliX, Inc., VENDYS vascular reactivity measurement.
Angioplasty/Stents mechanical interventions. Create more endothelial dysfunction 
Autonomic nervous system is the auto regulatory mechanism that coordinates all the involuntary functions in the body including all smooth muscle activity. It is a dynamic system that maintains a constant basal capillary tone at half maximal stimulation of the SNS. This is a critical phenomenon that lends dynamicity and rapid response capability for the ANS. Systemic vascular resistance and Pitch Tone are classic examples for autonomic dynamicity. Pitch Tone as noted above is maintained by a fine balance between SNS and PSNS input. Pitch Tone is essential for proper functioning of the circulatory system and maintenance of blood pressure. Alterations in the Pitch Tone cause reflex brainstem hypothalamic stimulation. Hypothalamus is a key control center of the limbic system and along with central vagal nucleus forms the hypothalamic - central vagal axis that coordinates several brain stem nuclei and centers such as cardiovascular center and respiratory center. This is the central clinical autonomic loop at the brain stem that generates various involuntary and reflex physiologic responses by various organ systems throughout the body (Figure-4). These responses maintain body homeostasis, hormone regulation, fluid/electrolyte and temperature regulation via balanced autonomic nervous system input. Hypothalamus is also the center for emotional responses and stress reactions. Therefore, stress induced cardiovascular disease and co-morbid conditions are due to abnormal central clinical autonomic loop function and dysautonomia.

Dysautonomia vasculitis, in summary, is triggered by vascular endothelial malfunction due to dysfunctional autonomic regulation of the vascular endothelium and GAP junctions. (Figure-7) [18].

\section{CENTRAL CLINICAL AUTONOMIC LOOP of ADIRAJU(CCALA)}

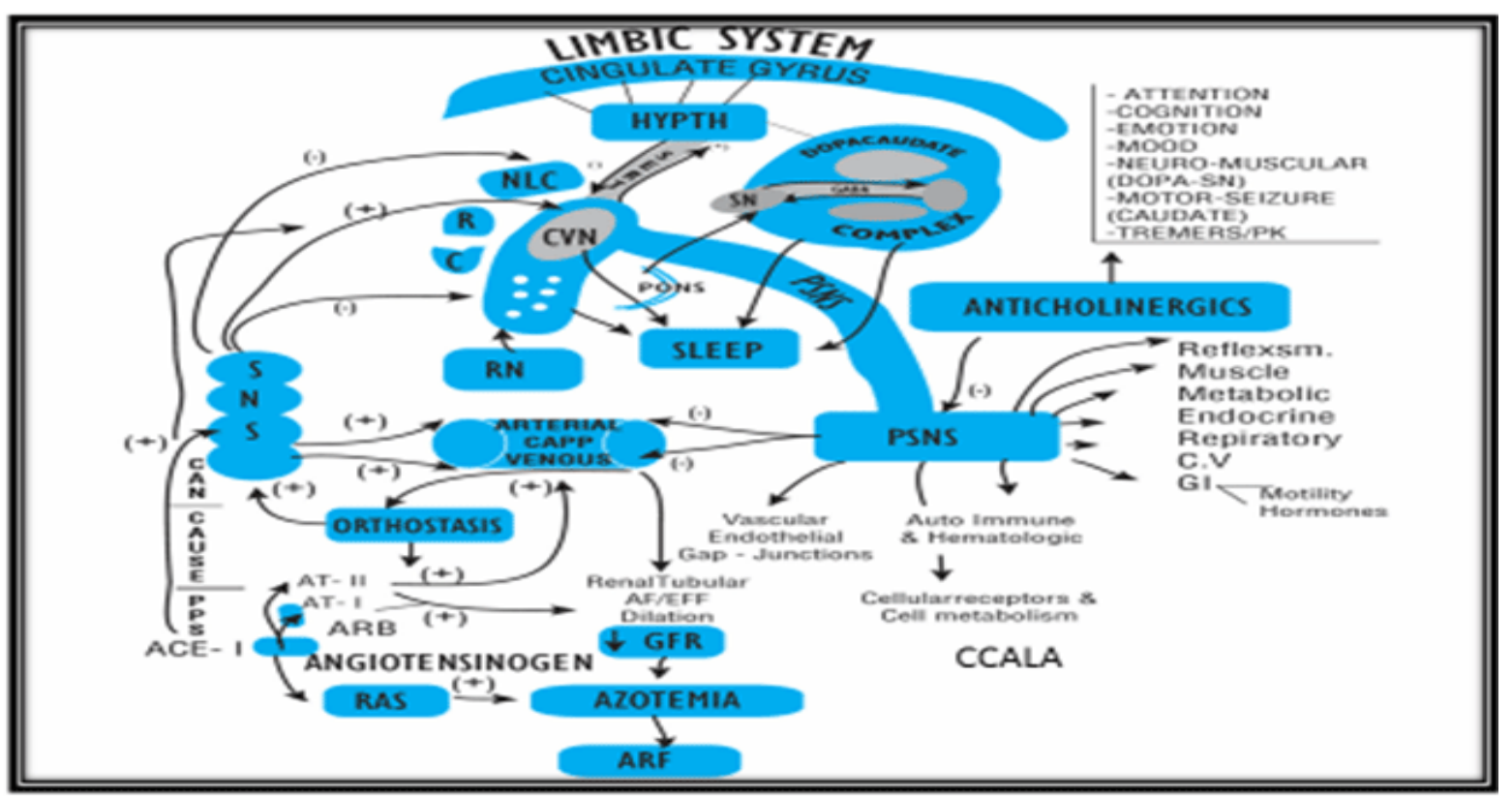

(C) Ramesh Adiraju All Rights Reserved

Figure 4: (graphic designing by Katrina Tapper)

\section{Dysautonomia and vasculitis:}

Vascular endothelium is unique, complex, and has multitasking functions. It achieves these functions through modulation of cellular GAP junctions (involuntary smooth muscle bands). Vascular endothelial functions include:

- Absorption of oxygen, nutrients, and essential hormones

- Maintain local vascular homeostasis

- Vasoreactivity and vascular inflammation control through nitric oxide synthesis and bioavailability (c-GMP pathway). Maintaining optimal balance between vascular antioxidants (catalase and super oxide dismutase) and pro-oxidative hormones and reactive oxygen species (endothelin, angiotensin II)

- Local Vascular Homeostasis (LVH) and coagulopathy through regulation of local hormones (PDGF, VDGF, and anticoagulants such as interleukins, prostaglandins - PGE, PGI, and prostacyclin)

- Protective functions - impermeable barrier to toxins and chemotaxis regulation to prevent vascular inflammation through GAP junction modulation. Endothelial integrity, vasoreactivity, and GAP junction activity are regulated by balanced reflex SNS and PSNS input to the vascular system. This is a centrally regulated process from brainstem CVN (central vagal nucleus) 
Abnormal vasoreactivity is a function of endothelial dysfunction commonly associated with atherosclerosis and T2DM. ANSD (autonomic nervous system dysfunction) causes systemic vascular inflammation and hypercoagulable state by triggering endothelial dysfunction. There is increasing evidence for inflammatory etiology for atherosclerosis with inflammatory markers - hsCRP and homocysteine demonstrated in atherosclerosis [14]. Dysautonomia is the underlying etiology for T2DM and endothelial dysfunction. Restenosis in diabetic vasculopathy is triggered by increased inflammation with more fibrosis and less hyperplasia. Increased pro-inflammatory cytokines (TNF-a, IL-1b) trigger endothelial dysfunction with vascular inflammation and hypercoagulable state. Increased PAI-I expression and suppression of tPA are also seen with diabetic vasculopathy that predisposes to hypercoagulable state. These alterations in LVH are triggered by dysautonomia. Endothelial Integrity, vasoreactivity and Gap junction activity are regulated by balanced reflex SNS and PSNS input to the vascular system. This is a centrally regulated process by the brainstemCVN (central vagal nucleus) and hypothalamic axis (Figure-4).

Autonomic system with sympathetic and parasympathetic systems along with brainstem CVN (parasympathetic center) and the hypothalamus (physiologic sensory input center) and other brainstem nuclei constitutes the auto regulatory system CCALA (Figure. 4). There is no sympathetic center in the brainstem. However, sympathetic neurotransmitter norepinephrine crosses the blood brain barrier and has a reflex feedback stimulation of the CVN. This is the only muscarinic sympathetic receptor. This feedback sympathetic stimulation of the CVN completes the CCALA. Sympathetic system is therefore reactive/reflex (fight and flight)-catabolic, while parasympathetic with CVN is a midline brainstem structure and has regulatory responses and controls the sympathetic functions.

CentralClinicalAutonomicLoop ofAdiraju(CCALA) (ref-18)comprises of a group of brain stem nuclei

(1) CVN at nucleus solitarius made of acetylcholine secreting giganto-cellular formation. This is a mid-line structure and has regulatory function by definition (Figure. 5).

(2) Nucleus locus ceruleus: Lateral nucleus with reflex reactive functions. Norepinephrine is the neurotransmitter.

(3) Raphe nuclei: central structure. Locally active medullary serotonin release. Contributes to regulate pain responses and sleep cycle.

(4) Hypothalamus: Central structure; diencephalic serotonin release; has multiple regulatory sub-nuclei. Key control center of the limbic system and central clinical autonomic loop.

(5) Basal ganglia: Substantia nigra; caudate complex; secrete various locally active neurotransmitters-GABA (GammaAminobutyric acid); endorphin/encephalin; reflex responses.

(6) Hippocampus/fornix: Secrete endorphin/encephalin; central structure; remote memory; emotional recall; coordinated by CVN and hypothalamus.

(7) Cardiovascular center: Lateral structure; coordinated by CVN.

(8) Respiratory center: lateral structure; coordinated by CVN and raphe nuclei.

(9) Autonomic nervous system: Sympathetic and parasympathetic systems; abnormal peripheral capillary tone triggers CCALA that causes ANS dysfunction inducing autonomic resistance.

Autonomic resistance predisposes to systemic disease states of various systems-Cardiovascular/hormonal/immune systems with psycho-somatic manifestations and stress reactions due to hypothalamic involvement.

Abnormal vasoreactivity is a function of endothelial dysfunction commonly associated with T2DM. Several studies have linked T2DM and vasoreactivity to endothelial dysfunction [5]. However, a recent study (Yong-Jian Li, SeungWoon Rha - Cardiovascular center, Korea University, Gurogu, Seoul, Korea) showed no association of T2DM with CAS (coronary artery spasm), suggesting the existence of a different mechanism for CAS (abnormal vasoreactivity) and CAD (J Invasive Cardiology,2014;26(6):234-239). Dysautonomia is the underlying etiology for T2DM and endothelial dysfunction leading to diabetic vasculitis and atherosclerotic vascular disease progression [18]. Hyperlipidemia is only a contributing factor that causes plaque formation only in a setting of endothelial dysfunction and vascular inflammation. Therefore, stabilization of endothelium and controlling systemic inflammation are the key components for a cost-effective and successful longterm solution in management of vascular disease and type 2 diabetes with diabetic vasculopathy. We believe autonomic nervous system dysfunction is the common underlying pathophysiology for both these processes and other associated comorbidities such as sleep apnea, metabolic syndrome, secondary hypertension and renal insufficiency (Figure-7). Atherosclerosis is initiated by retention of atherogenic lipoprotein particles that trigger maladaptive inflammatory response in the vascular wall. Oxidative damage, endothelial dysfunction, and activation of immune responses through interleukins and prostaglandins lead to vascular atherosclerosis. All these processes are triggered by dysautonomia (ANSD cascade). Autonomic system regulates vascular wall immune responses and c- GMP pathways that maintain Nitric Oxide (NO) concentration. NO induces vasodilatation and endothelial stabilization to maintain vas0reactivity and oxidative free radical concentration.

Autonomic system is the auto regulatory system in the body that affects all the involuntary and reflex mechanisms in our body. Auto regulatory system maintains body homeostasis (fluid and electrolyte balance and temperature regulation) and replenishes the essential physiologic functions and sustains various organ 


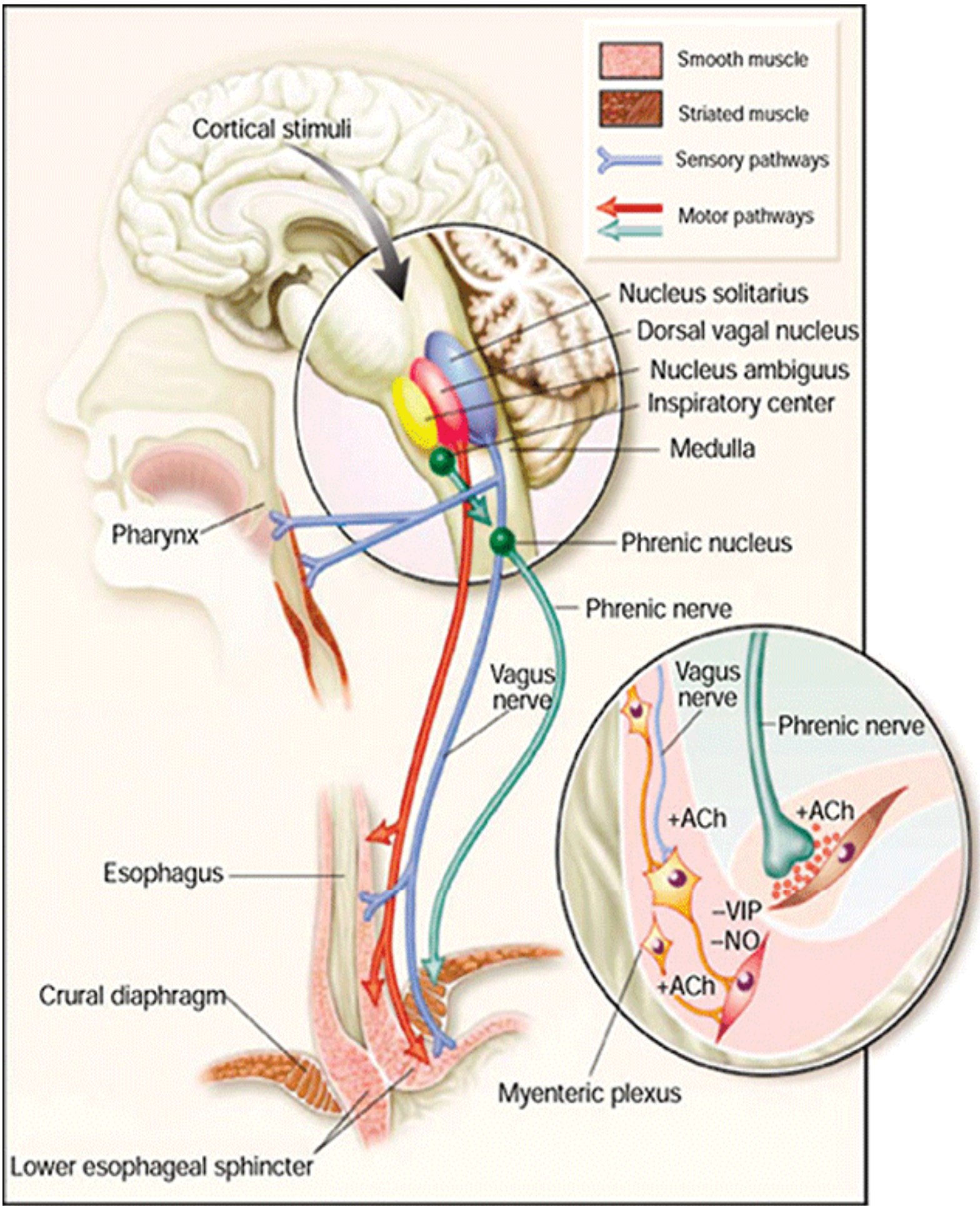

Figure 5: Central vagal nucleus location, midline at brainstem nucleus solitarius. Abbreviations: Ach, acetylcholine; VIP, vasoactive intestinal peptide; NO, nitric oxide. Courtesy: R.K. Mittal, R.K. Goyal, GI motility online (2006), doi:10.1038/gimo14. Nature Publishing Group.8. 
systems - cardiovascular system, hormonal regulation and responses, vascular integrity, liver enzyme regulation (cytochrome P450, etc.) metabolism, gastro-intestinal motility, and hormones. Heart beat and breathing are also maintained by CVN through regulating the brainstem cardiovascular and respiratory centers. Sleep cycle is also regulated by this system through complex interactions between brainstem nuclei via CCALA. Properly regulated sleep is an essential physiologic process during which parasympathetic surge maintains sleep cycle - REM and NREM sleep. During sleep cycles parasympathetic stimulation triggers physiologic replenishment of hormones and various organ systems. Dysautonomia results in abnormal sleep regulation and metabolic derangement. Dysautonomia also triggers leptin resistance. Leptin resistance is believed to be the underlying etiology for insulin resistance. Elevated plasma leptin levels and increased human soluble leptin receptor (hsLR) concentrations have been noted in T2DM patients. Hyperleptinemia in turn promotes hyper-sympathetic state and hypercoagulable state by expression of functional leptin receptors on platelets. Hyperleptinemia promotes vascular smooth muscle proliferation, atherogenesis, and restenosis. Leptin resistance triggers insulin resistance. Clinical manifestations of dysautonomia can be multi-system involvement with psychosomatic manifestations due to hypothalamic involvement in the CCALA. Stress induced cardiovascular disease and T2DM are therefore consequences of dysautonomia.

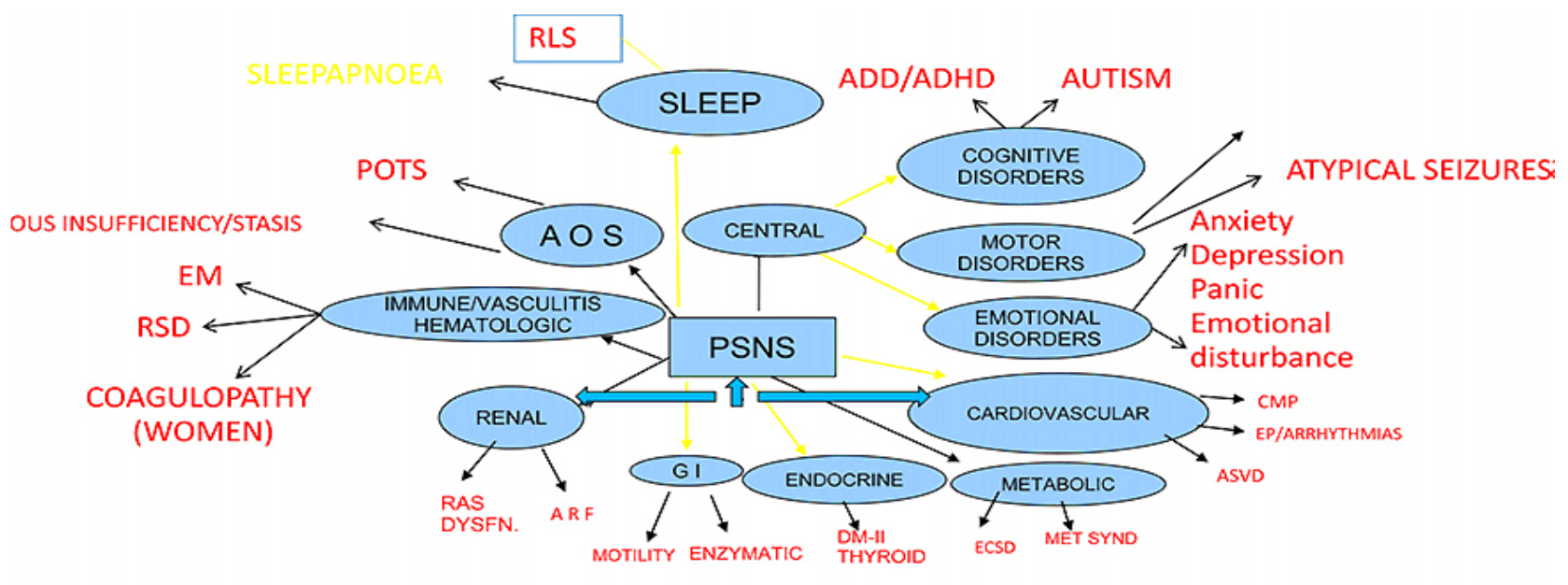

CLINICAL MANIFESTATIONS OF DYSAUTONOMIA

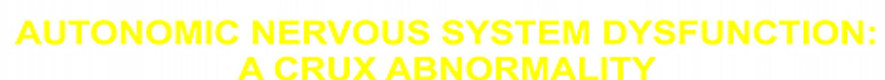

(c) Ramesh Adiraju. All Rights Reserved.

Figure 6: Abbreviations: PSNS, parasympathetic nervous system; AOS, autonomic orthostasis; RLS, restless leg syndrome; ADD/ADHD, attention deficit disorder/hyperactive disorder; RSD, reflex sympathetic dystrophy; EM, erythromyalgia; POTS, postural orthostasis tachycardia syndrome; RAS, renin angiotensin system; ARF, acute renal failure.

\section{Conclusions}

Behavioral and motivational mechanisms of the brain are regulated by the limbic system. Hypothalamus is the key control center. Hypothalamus has two-way communication with limbic system and central vagal nucleus (CVN). Hypothalamus sends signals upwards to thalamus and cerebrum, and downwards to reticular activating system and CVN. Serotonin is the neurotransmitter for hypothalamus and acetylcholine for CVN. Central brainstem structures have regulatory functions while lateral structures have reflex responses. The body's interaction with the environment and consequent physiologic responses are influenced by our emotions (mind/body complex). Therefore, stress induces disease while Transcendental Meditation, relaxation techniques (Figure-7), and exercise improve health. Bi-pedal posture of human evolution mandates optimal peripheral A-V capillary tone called "The Pitch Tone." Animals are predominantly sympathetic i.e. reactive. Humans, on the other hand, exercise forethought and cognizance prior to response. This is a higher intellectual function that requires coordinated controlled regulation that is achieved by the CCALA. Therefore, there is an element of psychological stress that is inherent with this response that can cause physiologic stress to the body. Balanced SNS and PSNS input is required to maintain Pitch Tone. Integrity of peripheral venous (calf pump) system and optimal Pitch Tone are therefore essential functions to maintain the integrity of CCALA and optimal responses of the auto regulatory system in the body. 


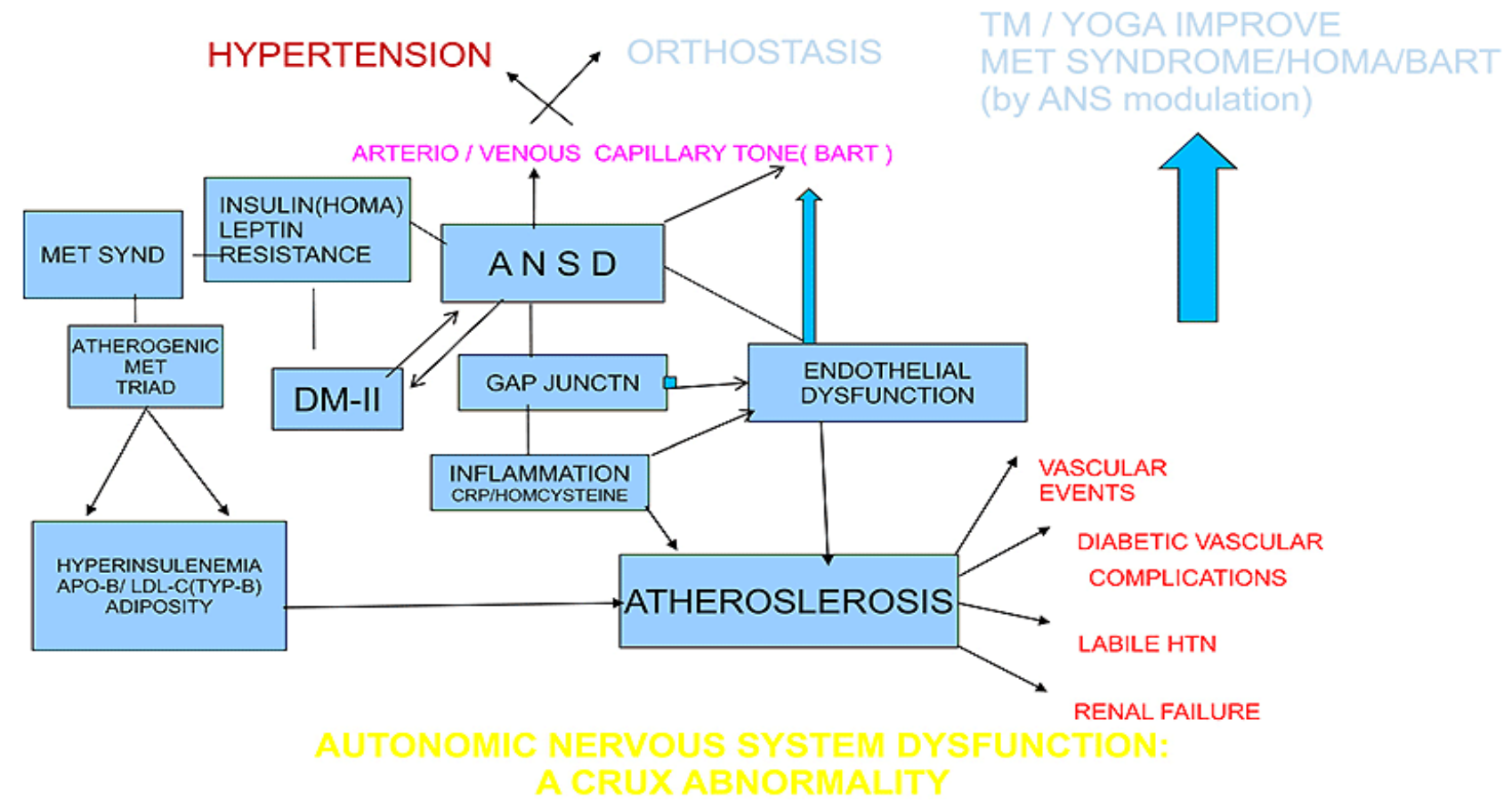

(c) Ramesh Adiraju. All Rights Reserved.

Figure 7: Abbreviations: ANSD, autonomic nervous system dysfunction; TM, transcendental meditation; HOMA, homeostasis model assessment; BART, brachial artery reactivity testing.

ANS through vasa nervosa and CCALA achieve this goal by maintaining a fine balance between SNS and PSNS- this is ANS coherence. "What maintains the circadian rhythm in our body, fight-n-flight, sleep/wakeful state, excitement/calm responses"it is the autonomic nervous system. Sympathetic (SNS) and parasympathetic (PSNS) systems constitute the autonomic system [18]. A critical balance between SNS and PSNS input is essential to maintaining optimal functioning of the various organ systems of the body. This balance is achieved through a process called resonance. Resonance is a dynamic balance between SNS and PSNS. PSNS is the regulator while SNS is the reactor. Autonomic resonance is, in turn, regulated by hypothalamic trigger and complex interaction sequence between various brainstem nuclei that constitute CCALA. CVN controls the CCALA and interacts with the hypothalamus to coordinate emotional behavior, sleep cycle, cognitive functions, and body physiology. CVN regulates and counter balances SNS activities to regulate various organ system functions and respiratory and cardiovascular centers. Hence, the close correlation observed between stress and emotions, with disease processes. Emotional input, depending on the type of emotion, and one's attitude towards the input, can either enhance or deplete one's energy reserve and response. Heart rate variability is a well-known measure of the sympathetic activity. Blunted heart rate variability is an indicator of loss of autonomic dynamicity that predisposes to cardiac arrhythmias and cardiomyopathy. Respiratory frequency is a function of the parasympathetic system. Respiratory rhythm is a powerful physiologic stimulus that has the capacity to alter several physiologic functions and emotional responses. Therefore, yoga and transcendental meditation with breathing techniques have demonstrated benefits in controlling diabetes, hypertension, and cardiovascular ailments [1]. Autonomic resonance along with proper coordination of the CCALA facilitates smooth flow of information between higher brain and body through autonomic system - this is autonomic coherence. Coherence at the CCALA facilitates proper physiologic responses to emotional input and appropriate dynamic stability of various organ systems. Autonomic resistance on the other hand is abnormal responses to external (erratic dietary habits, abusive habits such as smoking, environmental stress) or internal (from within the body from organ systems such as insulin receptor resistance, abnormal peripheral vascular tone, emotional stress, etc.) stimuli that result in distress and challenge the autonomic system leading to autonomic imbalance. This is the underlying cause for several disease processes [18]. Heart and vascular system, hormonal systems are particularly sensitive to autonomic resistance. Heart and vascular system is the primary responder to CCALA. The rest of the body follows the lead from the heart. "Hence our emotions are close to our heart."'Therefore, heart rhythm and cardiac autonomic balance is of paramount importance in maintaining autonomic resonance and CCALA coherence. Autonomic nervous system operates at varied frequency oscillations: VLF - very 


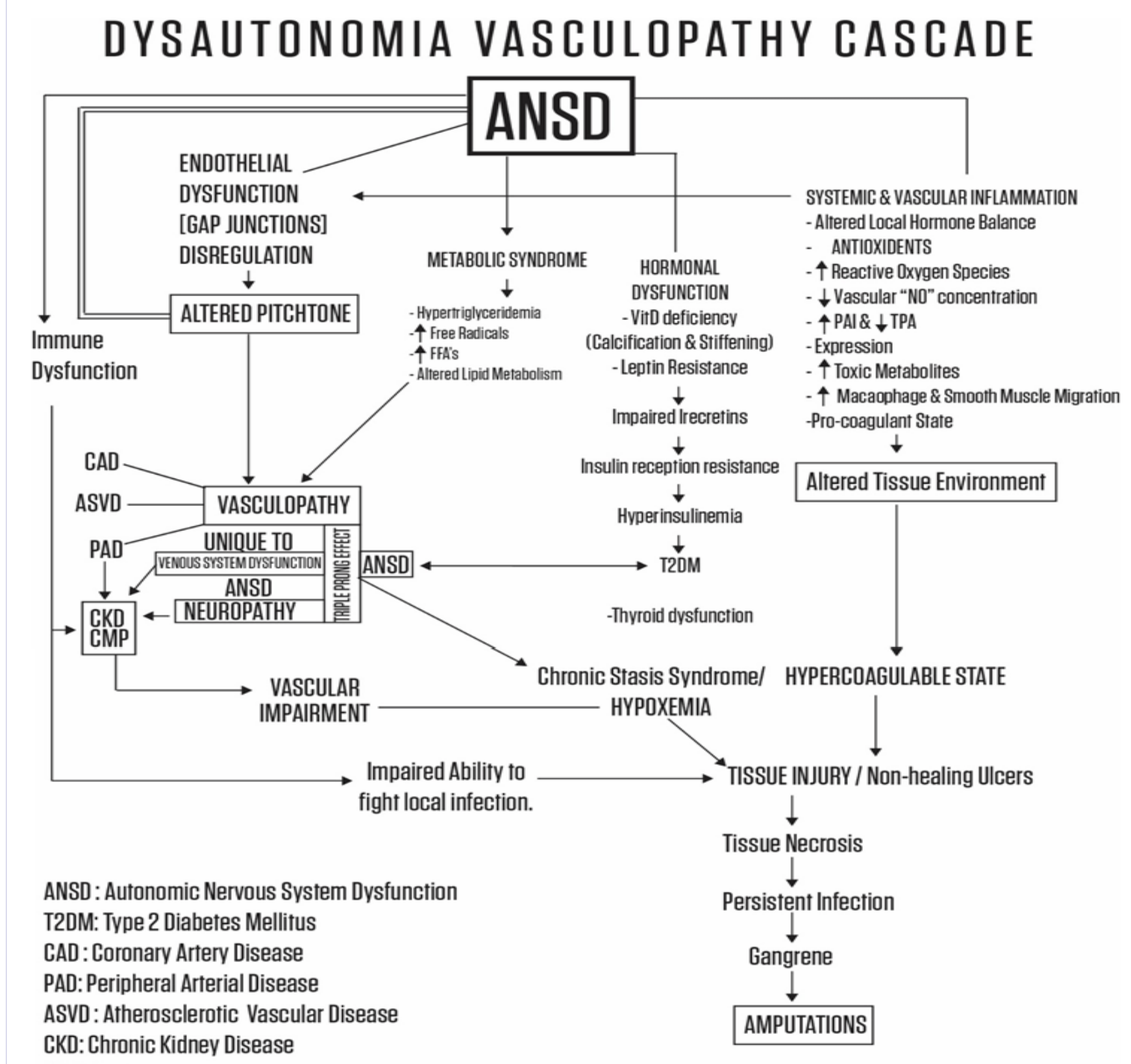

CMP: Cardiomyopathy

c. Ramesh Adiraju. All Rights Reserved

Figure 8:

low frequency oscillations $(0.001-0.04 \mathrm{~Hz})$ - Thermoregulation frequency. LF - low frequency oscillations $(0.04-0.15 \mathrm{~Hz})-$ SNS activity or sympathetic rhythm. HF - high frequency oscillations (0.15-0.4 Hz) - PSNS activity or parasympathetic rhythm. PSNS, its respiratory relationship and SNS regulation is demonstrated during young age when the autonomic dynamicity is maximum by sinus arrhythmia with deep breathing. This is facilitated by CVN overdrive over the cardiovascular center. Rapid shallow breathing on the other hand is SNS stimulation that induces rapid heartbeat. Sighing to relieve stress and anxiety is a reflex mechanism to trigger PSNS stimulation for relaxation. Forced short exhalations or rapid shallow breathing is sympathetically driven - the fight and flight response also called dog breathing, is triggered as a stress response (Panic attack) [3]. Demonstrated that Kapalbhati (a type of rapid shallow breathing technique) enhances low frequency oscillations - sympathetic rhythm, whereas altered breathing exercises involving deep inhalation have been shown to affect high frequency oscillations only but not LF oscillations suggesting PSNS stimulation only.

Physicians often follow disease model to organize patient symptoms and follow treatment modalities dictated by evidence 
data. Patients experience illness; physicians categorize it into a disease state. Modern medicine is unique in that the focus is exclusively biomedical. This approach has a risk of losing patient confidence. Indigenous healing approaches take the patient into confidence, but do not take structural and pathophysiologic derangements into consideration. Patient confidence is essential for favorable response to treatment. The body's physiology has to reciprocate to treatment administered. This reciprocation is an internal physiologic response that is mediated by CVN - hypothalamic axis through CCALA. Dysautonomia triggers physiologic stress and hinders this process. It can also result in adverse reactions to treatment. Advancement in medicine is following the lead of the disease process manifestations such as elevated blood sugar, elevated cholesterol, high BP, systemic inflammation, and hormone imbalances. These are consequences of secondary hyper-sympathetic state triggered by pardoxic parasympathetic response. The crux of the problem is dysautonomia. Hormone imbalances trigger metabolic derangement and systemic inflammation due to parasympathetic dysfunction and loss of CCALA coherence. Elevated blood sugar and hypertriglyceridemia are secondary consequences. High intensity exercise and aggressive sugar control can be counterproductive by triggering hyper- sympathetic and paradoxic parasympathetic state. This has been demonstrated in recent clinical trials [9, 11]. Tight blood sugar control does not reduce the incidence, complications, and cardiovascular morbidity and mortality in diabetics. This was demonstrated in major clinical trials - BARIII trial, and recently in two major large population diabetic trials (ACCORD, ADVANCE) [9]. The incremental value to risk prediction (i.e. change in C-statistic) is virtually identical for hsCRP as for triglycerides (TC) and high density lipoprotein cholesterol (HDLC), both of which are universally measured in all global risk prediction models [2]. Transcendental meditation and relaxation techniques, and breathing exercises have been shown to benefit T2DM, hypertension, and coronary artery disease progression [1]. The underlying physiologic mechanism for these benefits is parasympathetic modulation, as breathing techniques and meditation (concentration) are regulated by central parasympathetic nucleus (CVN) at the nucleus solitarius along with hypothalamus. CCALA coordinates these responses. The underlying dysfunction is autonomic dysfunction with abnormal parasympathetic responses. Treatment should be directed at assessment and treatment of dysautonomia at the brainstem level, i.e. CCALA (Figure-9)

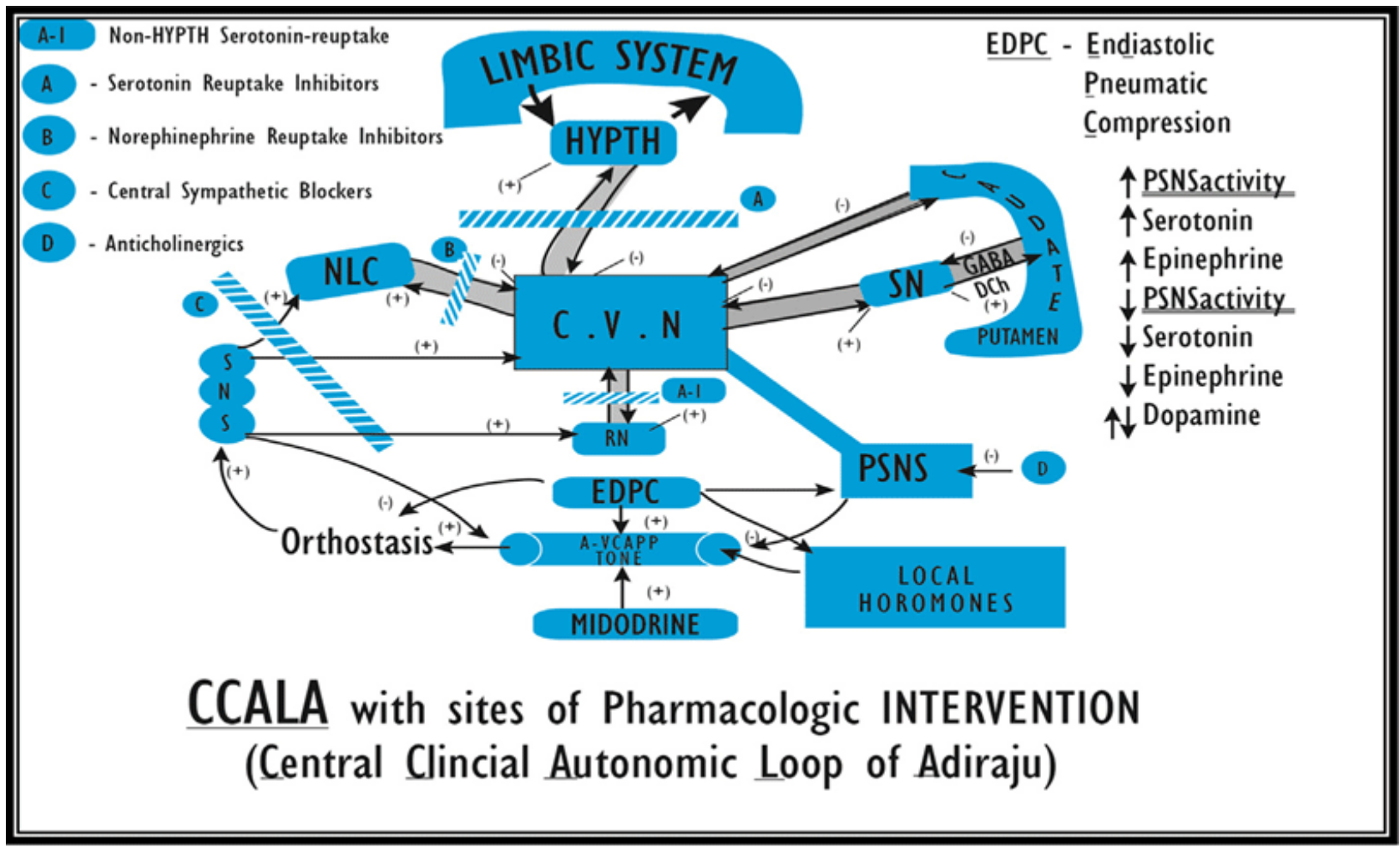

(c) Ramesh Adiraju. All Rights Reserved.

Figure 9: 


\section{References}

1. Paul-Labrador M, Polk D, Dwyer JH, Velasquez I, Nidich S, Rainforth $\mathrm{M}$, et al. Effects of a randomized controlled trial of transcendental meditation on components of metabolic syndrome in subjects with coronary heart disease. Arch Intern Med. 2006;166(1):1218-1224.

2. Emerging Risk Factor Collaborators. N Engl J Med. 2012; 367(14):1310-1320.

3. Raghuraj P, Ramakrishnan AG, Nagendra HR, Telles S. Effect of two related Yogic breathing techniques on heart rate variability. Indian J Physiol Pharmacology. 1998;42(4):467-472.

4. Kleinman A, Eisenberg L, Good B. Culture, illness, and care: clinical lessons from anthropologic and cross-cultural research. Ann Intern Med. 1978;88(2):251-258.

5. Lerman A, Zeiher AM. Contemporary review in cardiovascular medicine. Endothelial function: cardiac events. Circulation. 2005;111(3):363-368

6. Pinto G, Natalicchio H, Marchitti P. Physiology of incretins and loss of incretin effect in Type 2 diabetes and obesity. Arch Physiol Biochem. 2013;119(4):170-178.

7. Drucker DJ. Enhancing incretin action for the treatment of Type 2 diabetes. Diabetes Care. 2003;26(10):2929- 2940

8. Peter A. Incretin based therapies: review of current clinical trial data. Am J Med. 2010;123(March (3 suppl)):528-537.
9. Dluhy RG, McMahon GT. Intensive glycemic control in the ACCORD and ADVANCE trials - Editorial. N Engl J Med. 2008;358(June):2630-2638.

10.Vilsball T. On the role of the incretin forms GIP and GLP-1 in the pathogenesis of type- 2 diabetes mellitus. Danish Med Bull. 2004;51(4):364-370.

11. Colberg SR. The impact of exercise on insulin action in type 2 diabetes mellitus: relationship to prevention and control. Insulin. 2006;1:8598.

12. Mason RP, Jacob RF. Membrane micro domains and vascular biology: emerging role in atherogenesis. Circulation. 2003;107:2270-2273.

13. Adiraju RK, Glessman I, Colombo J. Preliminary clinical applications of the ANS-R1000. MESPE J. 2001;3:33-45.

14. Ridker PM. Testing the inflammatory hypothesis of atherothrombosis: scientific rationale for the cardiovascular inflammation reduction trial (CIRT). J Thromb Haemost. 2009;7(1):332-339.

15. Ridker PM. Evaluating novel cardiovascular risk factors: can we better prevent heart attacks? Ann Intern Med. 1999;130(11):933-937.

16. Mason RP. Jacob RF. Membrane microdomains and vascular biology: emerging role in atherogenesis. Circulation.2003;107:2270-2273.

17. Figueroa XF, Duling BR. Gap junctions in the control of vascular function. Antioxid Redox Signal. 2009 Feb;11(2):251-66. Doi: 10.1089/ars.2008.2117

18. Ramesh K. Adiraju. Dysautonomia Type 2 diabetes and vasculitis. DOI: 10. 1016/j.jicc.2015.10.023. 